\title{
THE EXPONENTIAL DECAY OF SOLUTIONS OF THE WAVE EQUATION IN THE EXTERIOR OF A STAR-SHAPED OBSTACLE
}

BY PETER D. LAX, CATHLEEN S. MORAWETZ AND RALPH S. PHILLIPS ${ }^{1}$

Communicated August 7, 1962

In this paper we study the behavior for large time of solutions of the wave equation in three space dimensions in the exterior of some smooth, bounded reflecting obstacle which is assumed to be starshaped. We shall prove that, given an initial disturbance, the bulk of its energy is propagated to infinity. The precise statement of the result is Theorem IV; it has as its corollary the following result:

Let $u$ denote a smooth solution of the above exterior problem whose initial values have finite energy and are zero outside of some bounded region. Then at a fixed point $x, u(x, t)$ decays exponentially with time.

When the scattering obstacle is a sphere, this result has been deduced by Wilcox [5] by analyzing the explicit expression for the solution obtained by separation of variables. In [4], C. S. Morawetz has proved that energy decays like the inverse square of time, and it follows that the solution decays like $1 / t$. The sharper result of the present paper is obtained by combining her result with the techniques developed in [1] and [2].

Theorem IV of this paper implies that the scattering matrix associated with the above problem can be continued analytically from the lower half-plane into a horizontal strip of the upper half-plane; a complete discussion of this can be found in [2].

$H_{0}$ denotes the Hilbert space of Cauchy data $\phi=\left(\phi_{1}, \phi_{2}\right)$ defined in the entire three-dimensional space, normed by the energy norm:

$$
\|\phi\|^{2}=\int\left(\left(D \phi_{1}\right)^{2}+\phi_{2}^{2}\right) d x .
$$

$U_{0}(t)$ denotes the operator which relates the data at time $t$ to the data at time zero of solutions of the wave equation in all of space. $H$ denotes the subspace of data which vanish inside the obstacle and $U(t)$ denotes the operator which relates the data at time $t$ to data at time zero of solutions of the wave equation in the exterior of the obstacle which vanish on the obstacle for all time. $U_{0}(t)$ and $U(t)$ are unitary operators mapping $H_{0}$ and $H^{0}$, respectively, onto themselves (conservation of energy) and they form one-parameter groups.

1 Research by the last named author was sponsored by the National Science Foundation, contract NSF-G 16434. 
A solution $u$ of the wave equation is called incoming or outgoing if $u=0$ for $|x| \leqq \rho-t$ or $|x| \leqq \rho+t$, respectively; here $\rho$ is a number so chosen that the sphere of radius $\rho$ around the origin contains the obstacle. The set of Cauchy data at $t=0$ of incoming and outgoing solutions will be denoted by $D_{-}$and $D_{+}$, respectively. Clearly $D_{-}$and $D_{+}$are closed subspaces of $H$.

Theorem I. (i) D_ and $D_{+}$are orthogonal.

(ii) $U(t)$ maps $D_{+}$into itself for $t$ positive, $D_{-}$into itself for $t$ negative.

(ii) is an immediate consequence of the definition of $D_{+}$and $D_{-}$; the proof of (i) is harder and is based on

TheOREM II. A solution of the wave equation in all space-time which has finite energy and which is zero in the double cone $|x|<|t|$ is zero identically.

Denote by $K$ the set of vectors in $H$ which are orthogonal to both $D_{+}$and $D_{-}$, and denote by $P_{+}$and $P_{-}$the orthogonal projections onto the orthogonal complements of $D_{+}$and $D_{-}$.

TheOREM III. For $t$ positive the operators

$$
Z(t)=P_{+} U(t) P_{-}
$$

map $H$ into $K$ and form a one-parameter semigroup over $K$.

Theorem III is a consequence of Theorem I.

The role of the operator $P_{-}$is to filter out signals which are coming in from very far away and which would not reach the obstacle for a very long time. Disturbances which are orthogonal to the incoming solutions would be expected to start interacting with the obstacle immediately; the effect of this interaction is to scatter that portion of the wave which impinges on the obstacle and to convert it after a finite time ${ }^{2}$ into an outgoing wave. This intuitive picture suggests that the norm of the operator $Z(t)$ tends to zero as $t$ tends to infinity; this is indeed so and is the main result of this paper.

TheOREM IV. There exist positive constants $T$ and $k$ depending only on $\rho$ such that

$$
\|Z(t)\| \leqq e^{-k t} \quad \text { for } t>T .
$$

Since $Z(t)$ forms a semigroup of contraction operators, in order to

${ }^{2}$ The restriction that the obstacle be star-shaped plays a crucial role here: it prevents long lasting reverberations. 
prove its exponential decay it is sufficient to exhibit some value of $T$ such that the norm of $Z(T)$ is less than one. This is accomplished with the aid of the following estimate contained in [4]:

Let $w(x, t)$ be any solution of the exterior problem with finite energy whose initial values have compact support. Let $k$ be a number such that both the supports of $w(x, 0), w_{t}(x, 0)$ and the obstacle are contained inside the sphere $|x| \leqq k$. Then, given any $\gamma<1$ and any positive $\epsilon$ there exists a $T_{0}$ depending only on $k, \gamma$ and $\epsilon$ such that for all $t$ greater than $T_{0}$ the energy in $w$ at time $t$ contained inside the sphere $|x| \leqq \gamma T$ is less than $\epsilon$ times the total energy.

Details of proofs will appear in a forthcoming paper.

\section{REFERENCES}

1. P. D. Lax and R. S. Phillips, The wave equation in exterior domains, Bull. Amer. Math. Soc. 68 (1962), 47-49.

2. - Spectral theory for the wave equation, (to appear).

3. C. S. Morawetz, The decay of solutions of the exterior initial-boundary value problem for the wave equation, Comm. Pure Appl. Math. 14 (1961), 561-568. (1962).

4. - The limiting amplitude principle, Comm. Pure Appl. Math. 15, No. 3

5. C. Wilcox, The initial-boundary value problem for the wave equation in an exterior domain with spherical boundary, Abstract No. 564-20, Notices Amer. Math. Soc. 6, No. 7 (1959).

NEW YORK UNIVERSITY AND STANFORd UNIVERSITY 